\title{
Comparison of methods for broadband electromagnetic characterization of Molded Interconnect Device materials
}

\author{
C. Orlob, D. Kornek, S. Preihs, and I. Rolfes
}

Leibniz Universität Hannover, Institut für Hochfrequenztechnik und Funksysteme, Appelstr. 9A, 30167 Hannover, Germany

\begin{abstract}
Combining the Molded Interconnect Device technology with the Laser Direct Structuring technology exhibits the potential of designing electrical and mechanical components on three-dimensional surfaces to increase functionality, level of integration and to reduce costs. When taking advantage of this technology especially in the design of RF devices, a precise knowledge of the electromagnetic parameters of the MID material is required, as the complex permeability and permittivity strongly influence the device performance. At present time, these materials are not electromagnetically characterized in the RF frequency range. In this paper different methods are therefore presented and compared with respect to their potentials for broadband electromagnetic characterization of Molded Interconnect Device materials.
\end{abstract}

\section{Introduction}

The Molded Interconnect Device technology (MID) in combination with Laser Direct Structuring (LDS) offers the possibility of designing electrical and mechanical components on three-dimensional surfaces to increase functionality, level of integration and to reduce costs. For example, transmission lines, antennas, switches, and connectors can be integrated on carriers like the covers of cellular phones or the cases of exterior mirrors of a car. However, applying this novel technology to RF circuits implies the precise knowledge of the electromagnetic properties of the MID material. Especially for MID based development of antennas dedicated for different applications like IEEE 802.11 WLAN, IEEE 802.15.1 Bluetooth or IEEE 802.16 WiMAX it is necessary to determine the complex permittivity $\epsilon_{r}=\epsilon_{r}^{\prime}-j \epsilon_{r}^{\prime \prime}$ and complex permeability $\mu_{r}=\mu_{r}^{\prime}-j \mu_{r}^{\prime \prime}$ over a wide frequency range.

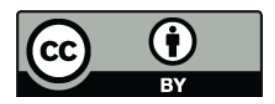

Correspondence to: C. Orlob (orlob@hft.uni-hannover.de)
Since these parameters are not quantified for RF frequencies at the present time, this paper presents a first characterization of an exemplarily chosen MID material Pocan DP T7140 LDS (Lanxess AG) demonstrating the features of the measurement methods, which were chosen with respect to the constraint of available samples geometries. Measurement principle, capability, bandwidth, uncertainties and sample requirements of each method are pointed out.

In Sect. 2 the known properties of the considered MID material are indicated and the MID LDS processing is shortly described. In the subsequent section the measurement methods are presented in detail followed by the measurement results shown and discussed in Sect. 4. The paper ends with a conclusion.

In the following, the investigated materials are generally categorized with the terms low permittivity, medium loss and low loss. The term low permittivity refers to materials where $\epsilon_{r}^{\prime} \leq 5$ and the terms medium loss and low loss correspond to $3 \times 10^{-4} \leq \tan \delta \leq 3 \times 10^{-2}$ and $\tan \delta<3 \times 10^{-4}$, respectively.

\section{MID LDS materials and processing}

This section gives information on relevant properties of the investigated material Pocan DP T7140 LDS and how it is applied in the MID LDS process. According to the data sheet, Pocan DP T7140 LDS is a solid polyethylene terephthalate/polybutylene terephthalate (PET/PBT) polymer with low permittivity and medium loss. In detail, a dielectric constant $\epsilon_{r}^{\prime}=4.1$ and loss factor $\tan \delta=\epsilon_{r}^{\prime \prime} / \epsilon_{r}^{\prime}=1.38 \times 10^{-2}$ are stated at $f=1 \mathrm{MHz}$.

Like any other MID LDS material it is provided with an additive in form of an organic metal complex (Schlueter et al., 2002). For usage in the MID LDS process, first its surface is activated by a laser beam as shown in Fig. 1. This separates the metal atoms from the organic ligands and roughens the surface enabling copper coating with a strong grip.

Published by Copernicus Publications on behalf of the URSI Landesausschuss in der Bundesrepublik Deutschland e.V. 


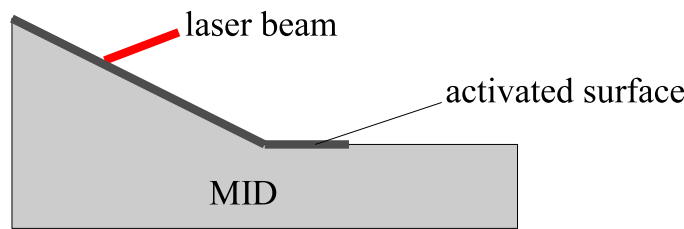

Fig. 1. MID LDS activation step.

Subsequently, the cleaning step follows, in which the debris from laser structuring is removed. Finally, the metallization is build up on the activated area by use of current-free copper baths. Depending on application, the copper layer can additionally be plated with gold or other metals by means of electroforming baths.

\section{Measurement methods}

In this section, the analyzed measurement methods are shortly presented in terms of functionality, measurement capability, frequency range of operation, measurement uncertainty and sample requirements. Further information about each method can be found in Clarke et al. (2002).

The first method considered is a commercially available admittance cell Agilent 16453A connected with the impedance analyzer Agilent E4991A. As shown in Fig. 2a, the dielectric is sandwiched by two electrodes forming a capacitor. The complex permittivity $\epsilon_{r}$ is determined as the ratio of the admittances for the material filled electrodes and for the air filled electrodes. This method can be used in the range from a few $\mathrm{MHz}$ to $1 \mathrm{GHz}$ and requires planar sheets with a thickness $0.3 \mathrm{~mm} \leq h \leq 3 \mathrm{~mm}$ and a diameter $d \geq 15 \mathrm{~mm}$. In accordance to the data specification of the impedance analyzer, a minimal uncertainty of approximately $\Delta \epsilon_{r}^{\prime} / \epsilon_{r}^{\prime}=7 \%$ can at best be achieved for measurement of low permittivity dielectrics.

The second technique refers to the coaxial probe HP $85070 \mathrm{~A}$, which is pressed against a flat surface of the dielectric, as shown in Fig. 2b. Assuming an isotropic, homogeneous and non-magnetic sample, the complex permittivity $\epsilon_{r}$ is derived from the reflection coefficient based on an quasi static model of the probe termination. Corresponding to the manufacturer's data, the theoretical operating range is $200 \mathrm{MHz} \leq f \leq 20 \mathrm{GHz}$, which is practically shortened by the effect of falling sensitivity with decreasing frequency. A minimum uncertainty of $\Delta \epsilon_{r}^{\prime} / \epsilon_{r}^{\prime}=5 \%$ in the upper part of the frequency range can be accomplished assuming appropriate contacting and calibration. This method requires samples with at least one flat surface and dimensions $h \geq 20 / \sqrt{\left|\epsilon_{r}\right|} \mathrm{mm}$ and $d>20 \mathrm{~mm}$.

The third method relies on on-wafer scattering-parameters measurement for coplanar waveguides (CPW) of different lengths (Arz and Leinhos, 2008). A CPW is formed from a signal conductor and a pair of groundplanes, all arranged a)

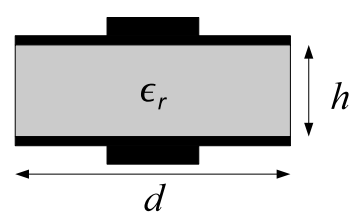

b)
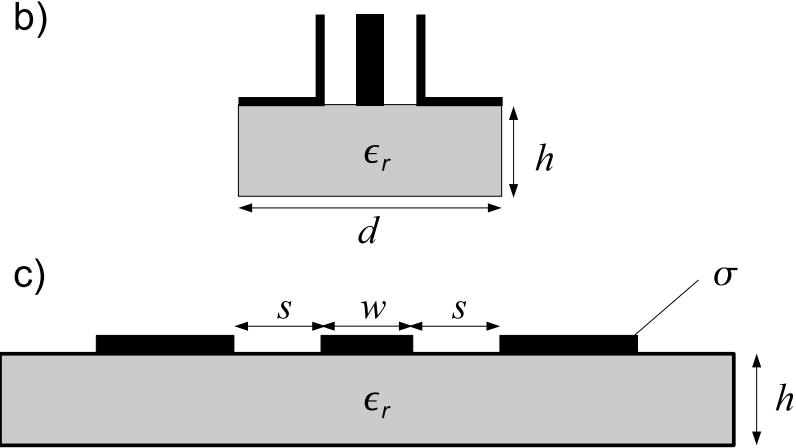

d)

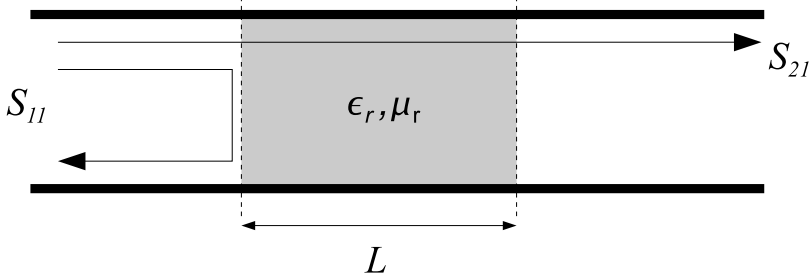

Fig. 2. Cross-sections of measurement setups: (a) admittance cell, (b) coaxial probe, (c) coplanar waveguide and (d) rectangular waveguide.

on top of the dielectric, as shown in Fig. 2c. Concerning the measurement procedure, first of all an effective propagation constant $\gamma_{\text {eff }}$ is determined from the measured raw data on the basis of a multiline-TRL algorithm (Marks, 1991). In a second step this result is used in a quasi-TEM model for coplanar lines (Heinrich, 1993), which assumes an isotropic, homogeneous and non-magnetic substrate material, to finnaly extract the complex permittivity $\epsilon_{r}$ of the substrate material. The chosen CPW dimensions should hold the condition $2(w+2 s)<h$ in order to approximately meet the assumption of an infinite thick substrate as demanded in the quasi-TEM model (Heinrich, 1993). For accurate loss factor extraction the model requires the value of the metallization's conductivity $\sigma$. This method covers a broad frequency range, whose lower limit depends on the maximal realizable length differences between the shortest and longest line and the upper limit is given by the measurement equipment like the vector network analyzer or the contact probes. A definite statement about the measurement uncertainty can not be issued. However, as shown in Arz and Leinhos (2008) this method exhibits a measurement accuracy comparable to the accuracy, which can be achieved with a split-cylinder resonator. 
The last method investigated is based on the rectangular waveguide technique. The sample, filling a section of waveguide of length $L$ as demonstrated in Fig. 2d, is electromagnetically characterized by the scattering-parameters

$S_{11}=\frac{\left(1-z^{2}\right) \Gamma}{1-(\Gamma z)^{2}}$

$S_{21}=\frac{\left(1-\Gamma^{2}\right) z}{1-(\Gamma z)^{2}}$

with the transmission coefficient

$z=e^{-\gamma L}$

and the reflection coefficient

$\Gamma=\frac{\frac{\mu}{\gamma}-\frac{\mu_{0}}{\gamma_{0}}}{\frac{\mu}{\gamma}+\frac{\mu_{0}}{\gamma_{0}}}$.

The terms $\gamma_{0}$ and $\gamma=f\left(\epsilon_{r}, \mu_{r}\right)$ stand for the waveguide propagation constants of the fundamental mode $\mathrm{TE}_{10}$ in air and material, respectively. In comparison with the other three discussed techniques this method features the capability to measure the relative permittivity as well as the relative permeability by solving the system Eqs. (1)-(2) via the Nicolson-Ross solution (Nicolson and Ross, 1970). In the case of measuring long samples this approach leads to the drawback of instability at frequencies corresponding to integer multiples of one-half wavelength in the material. Alternatively, when a non-magnetic material can be assumed the permittivity can be determined in a stable way by solving Eq. (2) numerically according to the procedure of BakerJarvis et al. (1990). In both approaches this method covers a frequency range of a single waveguide band, which is in this paper $8.4 \mathrm{GHz} \leq f \leq 12 \mathrm{GHz}$, and requires samples, which should be precisely matched to the inside dimensions of the waveguide to avoid air gaps. In this paper for the numeric solution a differential uncertainty analysis accounting for uncertainties in scattering-parameters and sample length $L$ is accomplished. In accordance with Baker-Jarvis et al. (1990), the total uncertainty is defined as

$$
\begin{aligned}
& \Delta \epsilon_{r}^{\prime}= \\
& \quad \sqrt{\left(\frac{\partial \epsilon_{r}^{\prime}}{\partial\left|S_{21}\right|} \Delta\left|S_{21}\right|\right)^{2}+\left(\frac{\partial \epsilon_{r}^{\prime}}{\partial \Theta_{21}} \Delta \Theta_{21}\right)^{2}+\left(\frac{\partial \epsilon_{r}^{\prime}}{\partial L} \Delta L\right)^{2}}
\end{aligned}
$$

where $\Delta\left|S_{21}\right|$ is the uncertainty in magnitude of $S_{21}, \Delta \Theta_{21}$ is the uncertainty in phase of $S_{21}$ and $\Delta L$ is the uncertainty in sample length $L$. The total uncertainty for the loss factor is defined respectively. For low permittivity samples with medium or low loss, uncertainties below $\Delta \epsilon_{r}^{\prime} / \epsilon_{r}^{\prime}=1 \%$ can be achieved for lengths $L$ longer than half the wavelength in the material. For this class of materials it can be expected by trend that the larger the sample length $L$ is, the lower the total uncertainty hence the higher the measurement reliability will be (Baker-Jarvis et al., 1990).

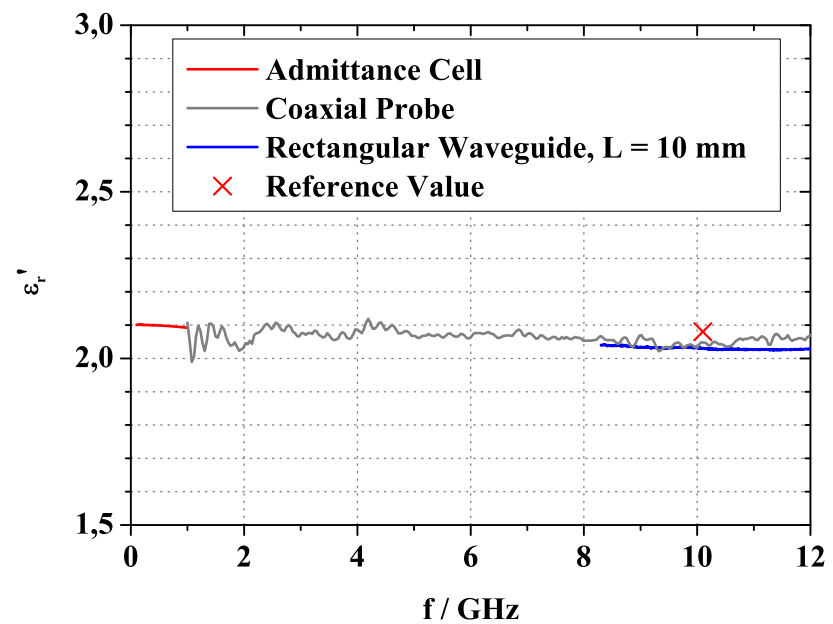

Fig. 3. Real part of the relative permittivity $\epsilon_{r}^{\prime}$ of Teflon.

\section{Measurements and comparison}

Except for the measurements utilizing the admittance cell, all measurements were carried out on the basis of a vector network analyzer (VNA). Before measuring, the admittance, coaxial probe and the rectangular waveguide setup were calibrated.

For the CPW measurements five lines with lengths between $2 \mathrm{~mm}$ and $55 \mathrm{~mm}$ were structered on a sheet of Pocan DP T7140 LDS with a thickness $h=2 \mathrm{~mm}$ by the use of the MID LDS technology. The CPW dimensions were chosen to $w \approx s \approx 300 \mathrm{um}$, which hold the required condition $2(w+2 s)<h$ for the assumption of an infinite thick substrate. The lines were contacted via probes and a separate DC measurement for the determination of the conductivity $\sigma$ was performed.

For the analysis of the measurement results achieved with the rectangular waveguide setup, the appearance of higher order modes, wall losses and air gaps between sample and waveguide walls were neglected. The uncertainties were calculated according to Eq. (5) for the given scatteringparameters uncertainties of the VNA and an estimated uncertainty in specimen length of $\Delta L=50 \mu \mathrm{m}$.

Furthermore, the admittance cell and coaxial probe methods were only used for the determination of the real part of the permittivity $\epsilon_{r}^{\prime}$, because of their low accuracy in loss measurements for an expected medium loss.

Before characterizing a MID material with almost unknown electromagnetic properties the performances of the admittance cell, the coaxial probe and the rectangular waveguide were tested by measuring $\epsilon_{r}^{\prime}$ of the reference material Teflon (PTFE). The results and a reference value from Krupka et al. (1998) are shown in Fig. 3. The corresponding uncertainties for the admittance cell, coaxial probe and rectangular waveguide are $\Delta \epsilon_{r}^{\prime} / \epsilon_{r}^{\prime}=14 \%, \Delta \epsilon_{r}^{\prime} / \epsilon_{r}^{\prime}=5 \%$ for $f \geq 9 \mathrm{GHz}$ and $\Delta \epsilon_{r}^{\prime} / \epsilon_{r}^{\prime}=1 \%$ respectively. The measurement 


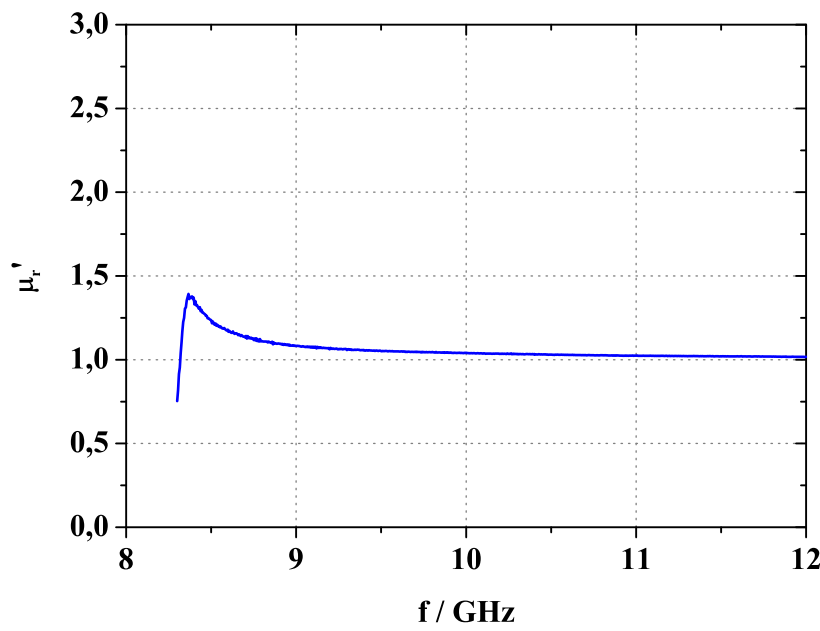

Fig. 4. Real part of the relative permeability $\mu_{r}^{\prime}$ as determined by the rectangular waveguide method using Nicolson-Ross solution.

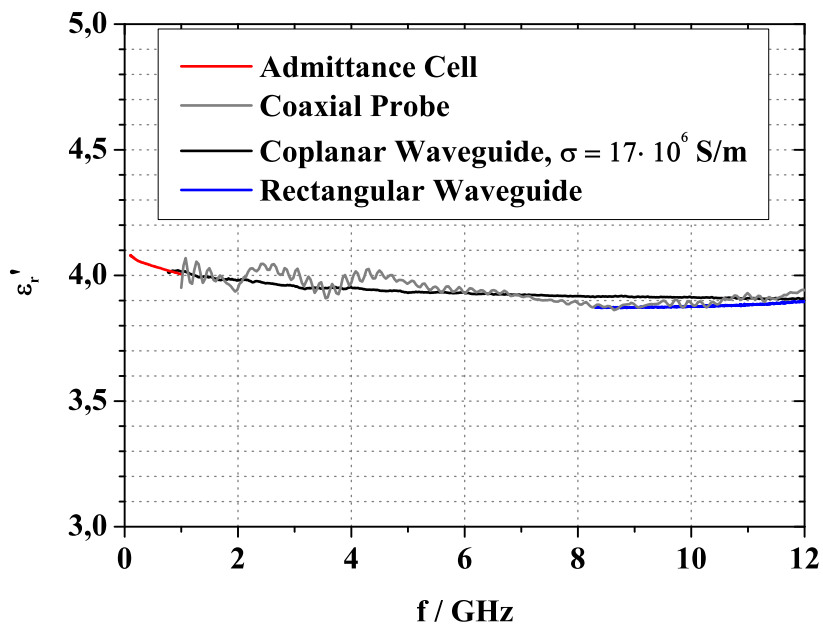

Fig. 5. Measured real part of the relative permittivity $\epsilon_{r}^{\prime}$ of Pocan DP T7140 LDS.

curve achieved with the coaxial probe shows in comparison to the other curves significant ripples in this frequency region due to low accuracy at low frequencies. However, all measurement results lie in the typical range for teflon $2 \leq \epsilon_{r}^{\prime} \leq 2.1$ and differ less than $5 \%$ from the reference value implying a reasonable performance of each method.

Based on this, the MID material Pocan DP T7140 LDS, which was assumed to be isotropic and homogeneous, was measured with all four methods. Since this thermoplastic includes an organic metal complex for LDS processing, it was first checked if the added complex caused a significant permeability. The result calculated with the NicolsonRoss solution for a sample with a maximal available length of $L=10 \mathrm{~mm}$ is displayed in Fig. 4. Except for lower frequencies near $f=8.5 \mathrm{GHz}$, where $\lambda / 2$-resonance occurs and the Nicolson-Ross solution fails, the condition $\mu_{r} \approx 1$ holds.

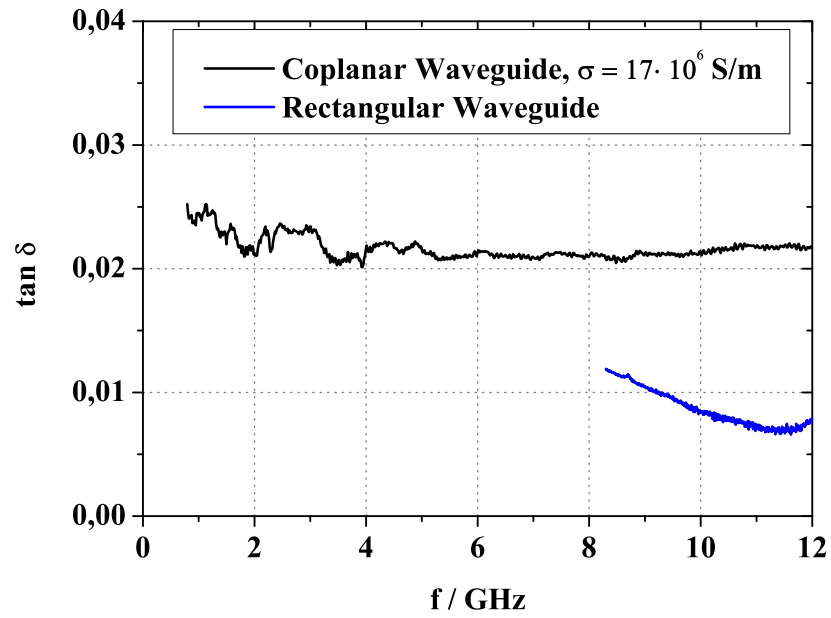

Fig. 6. Dielectric loss tangent $\tan \delta$ of Pocan DP T7140 LDS measured with both waveguide methods.

Consequently, the further analysis could focus on the determination of the complex permittivity only. The $\epsilon_{r}^{\prime}$ results determined with all four methods are presented in Fig. 5. In the case of the rectangular waveguide method the result was calculated with the numeric solution of Eq. (2) as described in the previous section. The yielded uncertainties for the admittance cell, coaxial probe and rectangular waveguide are $\Delta \epsilon_{r}^{\prime} / \epsilon_{r}^{\prime}=13 \%, \Delta \epsilon_{r}^{\prime} / \epsilon_{r}^{\prime}=5 \%$ for $f \geq 7 \mathrm{GHz}$ and $\Delta \epsilon_{r}^{\prime} / \epsilon_{r}^{\prime}=1 \%$, respectively. In particular, the results achieved with the admittance cell, CPW and rectangular waveguide method show a considerable mutual consistency indicating a permittivity $\epsilon_{r}^{\prime}$, which continuously falls from approximately 4 at $f=1 \mathrm{GHz}$ to approximately 3.9 at $f=12 \mathrm{GHz}$. By contrast the coaxial probe measurement curve shows the strongest variations for frequencies below $f=7 \mathrm{GHz}$. This could be a result of low accuracy as well as imperfect contact between the probe and the hard sample of Pocan DP T7140 LDS.

In addition, the loss factor could be determined with both waveguide methods as shown in Fig. 6. Which of the both curves is closer to the true value can not be stated so far. On the one hand the rectangular waveguide method exhibits a mean uncertainty within the X-Band of $\Delta \tan \delta / \tan \delta \approx 50 \%$ and on the other hand the CPW loss measurement could be affected by the roughness of the laser structured surface, which is not implicated in the used quasi-TEM model. A final statement could be issued by measuring a longer sample with the rectangular waveguide method or an additional resonator method. Unfortunately both possibilities could not be realized so far, due to lack of adequate samples. Nevertheless, a loss factor $\tan \delta$ in the dimension of $10^{-2}$ can definitely be assumed. 


\section{Conclusions}

In this paper four measurement methods for the electromagnetic characterization of MID materials have been presented and compared with each other. Each method has been investigated in terms of measurement setup, functionality, bandwidth, uncertainties, sample requirements and finally measurement performance.

The admittance cell enables measurement of $\epsilon_{r}^{\prime}$ with highest uncertainty. Since it requires only little sample and setup preparation, it is a suitable method for a first, less time consuming permittivity characterization. The coaxial probe is also a simple technique, but with a capability of higher measurement bandwidth. It makes little demands on the sample, but larger on the user having to regard to appropriate contacting and calibration, which were the reasons for noticeable ripples in the measurement curves. It is also a convenient method for a first broadband valuation of $\epsilon_{r}^{\prime}$. The CPW measurements also exhibit an adequate possibility of broadband permittivity characterization, but they invoke high expenses in sample preparation and measurement complexity. Especially for MID LDS structured CPWs a surface roughness probably has to be taken into account for accurate loss determination. Finally, the rectangular waveguide method represents the most powerful of the four discussed methods concerning measurement capability. With the potential of determination of both the complex permittivity and the complex permeability it is well suited for a basic classification of a material with unknown electromagnetic properties. Furthermore for low permittivity materials with medium or low losses it is well suited for the determination of $\epsilon_{r}^{\prime}$, but not necessarily for loss characterization. For accurate measurements long samples with respect to the wavelength are required, which are not always available.

Next to the comparison of the measurement methods, the MID material Pocan DP T7140 LDS was characterized within a frequency range of $f=0.1 \mathrm{GHz}$ to $f=12 \mathrm{GHz}$ for the first time. Measurement results indicate a permeability $\mu_{r} \approx 1$, a dielectric constant falling from $\epsilon_{r}^{\prime} \approx 4$ to $\epsilon_{r}^{\prime} \approx 3.9$ and a dielectric loss factor $\tan \delta$ in the dimension of $10^{-2}$. Finally, by considering the facts that different samples of this material were measured with four methods, each having a different electromagnetic field configuration, and that the results in $\epsilon_{r}^{\prime}$ show a mutual consistence, the assumption of a homogeneous and isotropic material could be confirmed.
Acknowledgements. The authors wish to thank LPKF Laser \& Electronics AG, Garbsen for structuring the CPW substrate. We also thank Rosenberger Hochfrequenztechnik GmbH \& Co. KG for sponsoring the GSG Z-probes.

\section{References}

Arz, U. and Leinhos, J.: Broadband Permittivity Extraction from On-Wafer Scattering-Parameter Measurements, 12th IEEE Workshop on Signal Propagation on Interconnects, Avignon, France, 1-4, 2008.

Baker-Jarvis, J., Vanzura, E. J., and Kissick, W. A.: Improved Technique for Determining Complex Permittivity with the Transmission/Reflection Method, IEEE T. Microw. Theory, 38, 10961103, 1990.

Clarke, B., Gregory, A., Cannell, D., et al.: A Guide to the characterisation of dielectric materials at RF and microwave frequencies, National Physical Laboratory, Institute of Measurement and Control, London, 2003.

Heinrich, W.: Quasi-TEM Description of MMIC Coplanar Lines Including Conductor-Loss Effects, IEEE T. Microw. Theory, 41, 45-52, 1993.

Krupka, J., Derzakowski K., Riddle B., and Baker-Jarvis, J.: A dielectric resonator for measurements of complex permittivity of low loss dielectric materials as a function of temperature, Meas. Sci. Technol., 9, 1751-1756, 1998.

Marks, R. B.: A Multiline Method of Network Analyzer Calibration, IEEE T. Microw. Theory, 39, 1205-1215, 1991.

Nicolson, A. M. and Ross, G. F.: Measurement of the Intrinsic Properties of Materials by Time-Domain Techniques, IEEE T. Instrum. Meas., 19, 377-382, 1970.

Schlueter, R., Roesener, B., Kickelhain, J., and Naundorf, G.: Completely Additive Laser-Based Process for the Production of 3D MIDs, The LPKF LDS Process, 5th International Congress Molded Interconnect Devices, Erlangen, Germany, 2002. 